\title{
Structural insights on the effects of mutation of a charged binding pocket residue on phosphopeptide binding to $14-3-3 \zeta$ Protein
}

\author{
Sreevidya T S $\mathrm{S}^{1,3}$ | Somavally Dalvi ${ }^{2}$ | Prasanna \\ Venkataraman ${ }^{2,3}$ | Satyavani Vemparala1,3
}

\footnotetext{
${ }^{1}$ The Institute of Mathematical Sciences, C.I.T. Campus, Taramani, Chennai 600113, India

${ }^{2}$ Protein Interactome Lab for Structural and Functional Biology, Advanced Centre for Treatment, Research and Education in 6 Cancer, Sector 22, Kharghar, Navi Mumbai, Maharashtra, India 410210

${ }^{3}$ Homi Bhabha National Institute, Training School Complex, Anushakti Nagar, Mumbai 400094, India
}

Mutation of an invariant aspartate residue in the binding pocket of $14-3-3 \zeta$ isoform to alanine dramatically reduced phosphopeptide binding and induced opening of the binding pocket. Here we use extensive molecular dynamics simulations to understand the role of D124 residue in ligand binding. The simulations show that in the absence of phosphopeptide, the D124A mutation leads to binding pocket reorganization including widening up of the binding pocket at the major groove and repositioning of N173, a key residue that interacts with the main chain of phosphopeptide. These structural changes would interfere with the efficient binding of the peptide, corroborating the experimental observations. Both gain and loss of electrostatic interactions in the form of salt bridges strongly indicate a rearrangement of the network of interactions within the binding pocket. Limited proteolysis coupled mass spectrometry (lip-MS) of the apo and holo forms of WT and mutant protein shows a peptide binding helix otherwise buried in the WT protein was particularly accessible to trypsin in the apo form of the mutant protein and the region was mapped to 158-186 amino acid residues of $14-3-3 \zeta)$. These results further confirm the dynamic nature of D124A mutant. Unlike other basic residues, the invariant D124 facilitates peptide bind- 


\section{1 | INTRODUCTION}

14-3-3 proteins regulate multiple cellular process such as cell division, apoptosis, transcription, intracellular trafficking and cytoskeletal structure by interacting with many different client proteins $[1,2,3,4,5,6,7,8]$. 14-3-3 protein family consists of highly conserved $30 \mathrm{kDa}$ dimeric proteins that are present in all eukaryotic cells $[9,10]$. There are seven known 14-3-3 isoforms $(\beta, \gamma, \epsilon, \eta, \sigma, \tau$ and $\zeta)$ in mammals $[11,12]$. Their sequence and structures are largely identical and yet their functions are often non redundant $[13,14]$. The minor differences in sequence and structure of various isoforms may have a role to play in distinct functions even within the same pathway [15].

The heterogeneity and a sheer number of interacting partners of 14-3-3 proteins led to the speculation that there must be a common determinant for binding. The hypothesis was confirmed upon the identification of phosphoserine/phosphothreonine containing motifs present in many of the interacting partners [16, 17, 18, 19]. Peptide library screening studies led to the identification of two main 14-3-3 consensus motifs R-(S/Ar)-[+]-pS-[LEAM]- P (mode 1) and R- X-[Y/F]-[+]-pS-[LEAM]-P (mode 2).14-3-3 can bind to certain non- phosphorylated substrates such as Exoenzyme S, R18 peptides. The overall three dimensional structure of the isoforms are near identical [16, 10, 20]. Each monomer is composed of nine antiparallel alpha-helices $(\alpha \mathrm{A}-\alpha \mathrm{H})$. The helices labelled $\alpha \mathrm{A}, \alpha \mathrm{B}, \alpha \mathrm{D}$ form a dimer interface, whereas helices $\alpha \mathrm{C}, \alpha \mathrm{E}, \alpha \mathrm{G}, \alpha \mathrm{H}$ form a cup-shaped amphipathic client protein pocket. The positively charged residues such as K49, R56 and R127 interact with the phosphorylated serine of the ligand (residue numbering according to $\zeta$-isoform). Y128 stabilizes the incoming serine phosphate, maintaining the peptide in the requisite extended conformation. E180, V176, Y179 and W228 are involved in peptide side-chain interactions. The side-chains of N224, N173 and K120 form hydrogen bonds with the peptide's backbone. D124 lies at $3 \AA$ from N173 and R127 residues and mutating D124 to alanine results in loss of peptide binding, primarily due to structural alteration as deduced by biophysical parameters [21].

To fully comprehend the impact of D124A mutation, in this study, we have used extensive molecular dynamics simulations to understand the effect of D124A mutation on the structure of 14-3-3 and phosphopeptide binding. We modelled the structure of D124A apo and holo form (bound to mode-2 1QJA peptide) and performed simulations to capture the dynamic changes induced by mutation. While large scale global changes do not occur on D124A mutations, subtle and specific alteration in the position of a network of residues in the binding pocket provides an explanation for reduction in peptide binding. This is an important result as it distinguishes mutation induced structural changes from events that purely affect binding. Moreover, using LiP-MS we mapped the conformational changes in different regions of the WT and mutant apo proteins. To our surprise, we recover a unique fragment present only in the apo form of the mutant protein, indicating that this region is labile in the mutant substantiating our previous claim that the mutation leads to opening of the binding pocket. Sequence and structural alignment indicates that the segment belongs to the $\alpha$-helix located at the floor of the binding pocket. This observation also provides credence the hypothesis that, the small peptide will dissociate in the absence of stable interaction, while the large phosphopeptide/ phosphorylated protein can potentially remodel the flexible binding pocket necessary for stable binding. Thus by a series of orthologous techniques we provide important insights into the structure, protein interaction and conserved 


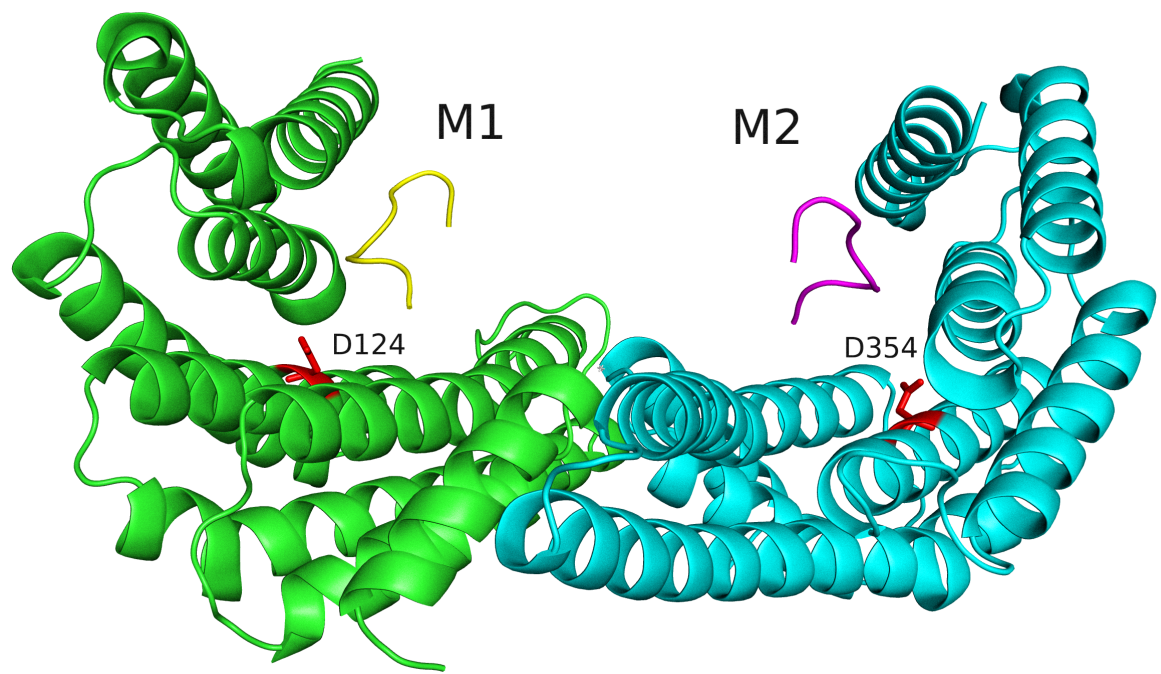

FIGURE 1 Cartoon representation of 14-3-3 WT $\zeta$ dimer system (PDB id: 1QJA) and the two monomers are referred to as $M 1$ and $M 2$. The relevant pocket residues whose mutations are considered in this study are labelled in orange (M1:D124, M2:D354) and the two bound phosphopeptides are shown in yellow (M1) and cyan (M2).

mechanism of binding and dissociation of this very important class of proteins.

\section{2 | METHODS}

\subsection{Molecular Dynamics Simulation:}

To understand the effect of D124A mutation on the binding of the phosphopeptide we simulate both the wild type

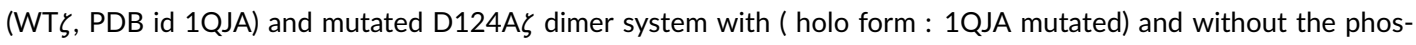
phopeptide (apo form : phosphopeptide removed from 1QJA). The details of all the systems studied are given in Fig. 2(a).The missing residues in the initial wild type PDB structure were modelled using Modeller software [22] and the correct protonation states of the ionizable residues were determined at the experimental pH of 6.2 (from the crystal structure) using DEPTH server [23] and appropriately changed before solvating the system. The dimeric form of 14-3-3 $\zeta$ with the phosphopeptide and the binding pocket residues mutated in this study are shown in Fig. 1.

Each system was solvated using TIP3P water model [24] and $150 \mathrm{mM} \mathrm{NaCl}$ salt was added to each system for overall charge neutralization and mimicking the physiological salt concentration. All the simulations were performed using Charmm36 forcefield [25, 26] in NAMD 2.12 software [27]. VMD software [28] and The PyMOL Molecular Graphics System, Version 2.4.2 Schrödinger, LLC [29] were used for visualisation and analyses were done using inhouse analysis codes using TCL scripting language. Energy minimization was done for 5000 steps using conjugate gradient method [30] and harmonic restraints were applied with force constant of $1 \mathrm{Kcal} / \mathrm{mol} / \AA^{2}$ on the complex for 1 ns with a time step of $1 \mathrm{fs}$ towards the equilibration of the system. The constraints were slowly released over $10 \mathrm{~ns}$ and all the systems were run for atleast 500 ns each with the total simulation of over 2 micro seconds. Langevin thermostat [31] was used to maintain constant temperature at 300K. Nose-Hoover Langevin piston [32, 33] with a 
decay period of $100 \mathrm{fs}$ and a damping time of $50 \mathrm{fs}$ was used to maintain pressure at $1 \mathrm{~atm}$. A cut-off distance of $12 \AA$ was used to calculate short-range van der Waals (VDW) interactions and the long-range electrostatics interactions was calculated by the Particle Mesh Ewald (PME) method [34, 35].

\subsection{Cloning, expression and purification of $14-3-3 \zeta$ WT and mutants:}

14-3-3 $\zeta$ WT and mutants D124A, N173V purified as described in Modi et al., [21].

\subsection{Fluorescence anisotropy:}

Fluorescence anisotropy (FA) was used to monitor the binding of FAM-RLYH(pS)LPA mode-2 1QJA (GL Biochem, Minhang, China) peptide to 14-3-3 WT and the mutant proteins. Various concentrations of 14-3-3 proteins in 50 $\mathrm{mM} \mathrm{Na}$-HEPES pH 8.0, $150 \mathrm{mM} \mathrm{NaCl}, 10 \%$ DMSO were incubated with $62.5 \mathrm{nM}$-labelled peptide, and the reaction was allowed to reach equilibrium for $30 \mathrm{~min}$ at room temperature in dark. The change in anisotropy was measured on Cytation 5, Biotek (Winooski, VT, USA). The G factor of the instrument was determined to be 1.2. Equilibrium KD was determined by fitting the data to a nonlinear regression model for single site-specific binding using GRAPHPAD PRISM 6 (GraphPad Software Inc, San Diego, CA, USA).

\subsection{Determination of $T_{m}$ :}

The thermal and chemical denaturation of the WT and the mutant 14-3-3 proteins were performed using nano-DSF (Prometheus NT.48) from Nanotemper Technologies (Munchen, Germany).The proteins at concentration of $15 \mu \mathrm{M}$ were used in $50 \mathrm{mM} \mathrm{Na}$ - HEPES buffer pH 8.0 containing $150 \mathrm{mM} \mathrm{NaCl}$ and $10 \%$ glycerol.

\subsection{Trypsin digestion:}

14-3-3 WT $\zeta$ and D124A $\zeta$ were reduced with $12.5 \mathrm{mM}$ DTT for $30 \mathrm{~min}$ at 37C, followed by alkylation with 40mM lodoacetamide for $45 \mathrm{~min}$ at $25^{\circ} \mathrm{C}$ in the dark. Further trypsin was added in 1:75 ratio (trypsin: protein) and incubated overnight at $37^{\circ} \mathrm{C}$. The trypsin digestion was stopped using formic acid. The peptide mixture was dried using speed vac. The dried pellet was resuspended into $0.1 \%$ formic acid. The peptide mixture was loaded onto desalting column and eluted with $80 \%$ acetonitrile. All the peptide samples were evaporated by using speed vac and redissolved in $0.1 \%$ formic acid followed by mass spectrometry [36].

\subsection{Mass spectrometry:}

The peptides were analyzed using Eksigent ekspertTM nano-LC 400 with cHiPLC $®$ system, with trap column (200 $\mu \mathrm{m}$ X $0.5 \mathrm{~mm}$ ) and analytical column $(75 \mu \mathrm{m}$ X $15 \mathrm{~cm})$, both packed with $3 \mu \mathrm{l}$ ChromXp C18 (120 Å). For reverse phase HPLC, with a gradient elution was performed for $60 \mathrm{~min}$. $0.1 \%$ FA in water and $0.1 \%$ FA in acetonitrile (ACN) served as solvent $A$ and $B$ respectively. Peptides were eluted with increasing percentage of Solvent $B$ at flow rate of $300 \mathrm{nl} / \mathrm{min}$. Eluated peptides were ionized using nanospray tip and analyzed in a positive ion mode on Triple TOF 5600+ mass spectrometer. Peptide analysis was done using software protein pilot TM software 4.5 and proteomics data Human + E. Coli downloaded on 2nd Jan 2019. Further analysis performed using Skyline software. 


\begin{tabular}{|c|c|c|}
\hline a) & Simulated System & $\begin{array}{l}\text { Simulation } \\
\text { time }\end{array}$ \\
\hline 1 & apo-WTל & $504 n s$ \\
\hline 2 & apo-D124AZ & $504 \mathrm{~ns}$ \\
\hline 3 & holo-WTל & $504 n s$ \\
\hline 4 & holo-D124A & $503 n s$ \\
\hline
\end{tabular}

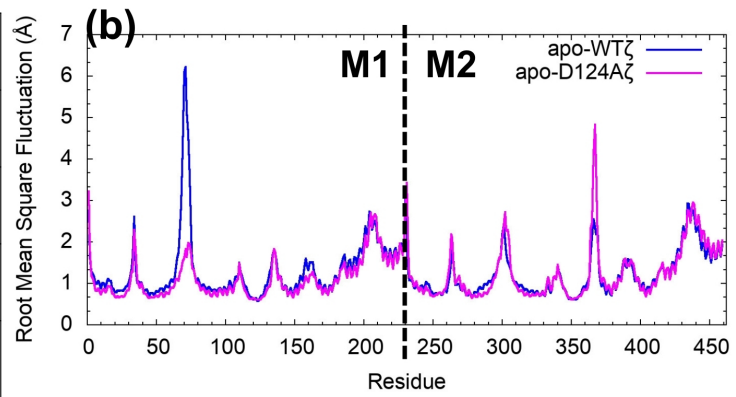

FIG URE 2 (a)Details of 14-3-3 $\zeta$ dimer systems simulated with (holo) and without (apo) the peptide. (b)RMSF of apo-WT $\zeta$ and apo-D124A $\zeta$ averaged over the last 100ns.

\section{3 | RESULTS}

To understand the effect of D124A mutation on the 14-3-3 $\zeta$ protein, analysis was first done on the apo-14-3-3 $\zeta$ trajectories. For convenience, chain $A$ is referred as $M 1$ and chain $B$ is referred as $M 2$ in the following results and discussion.

\subsection{Global conformational and dynamics changes in apo-14-3-3 $\zeta$ upon mu- tating D124 to alanine:}

To study the effect of D124A mutation on the binding of the phosphopeptide, the wild type (WT $\zeta$, PDB ID : 1QJA) and mutant D124A $\zeta$ dimer systems with (holo form: 1QJA mutated) and without the phosphopeptide (apo- form: phosphopeptide removed from 1QJA) were simulated. In the crystal structure (PDB ID: 1QJA) [16] of 14-3-3 protein there are missing residues and these include: Q67-Q76, A134-D136 in M1 and A134-D137 in M2 of the dimer, which are modelled in the present study (see Methods). We compared the RMS deviations over the length of the simulation, to probe for global structural changes. Residue level changes are shown in Fig. 2(b), illustrating the $C_{\alpha}$ Root Mean Squared Fluctuations (RMSF) as a function of residue number, averaged over the last 100ns. D124A mutation caused a change in fluctuations in the residue patches 62-80 in M1 and 358-372 (corresponds to 128-142 in M1) in M2 (Fig. 2(b)). The results suggest that D124A mutation does not induce any significant changes in the overall structure of the protein.

\subsection{D214A mutation alters conformation of binding pocket:}

Modi et al. [21], using various biochemical and biophysical approaches, have shown that D124A $\zeta$ protein has a more open structure than WT $\zeta$, resulting in loss of peptide binding. The opening up of structure was presumed to be due to local changes in the amphipathic groove upon mutating D124 to alanine. There are two openings in the binding pocket located at either end referred as major groove (M1=65 and 227, M2= 295 and 457) and minor groove (M1=40 and 209, M2= 270 and 439) at the either end of 14-3-3 $\zeta$ ((Fig. 4(b), (d)). Simulations reveal that major groove width significantly increases after D124A mutation, though minor groove width does not change much in $M 1$ monomer (Fig. 4(a,c)). However in M2 monomer, an increase in both minor and major groove width was observed (Figure S2(a,b)). The number of water molecules in the binding pocket before and after mutation were calculated in 
bioRxiv preprint doi: https://doi.org/10.1101/2021.09.27.461903; this version posted September 27, 2021.

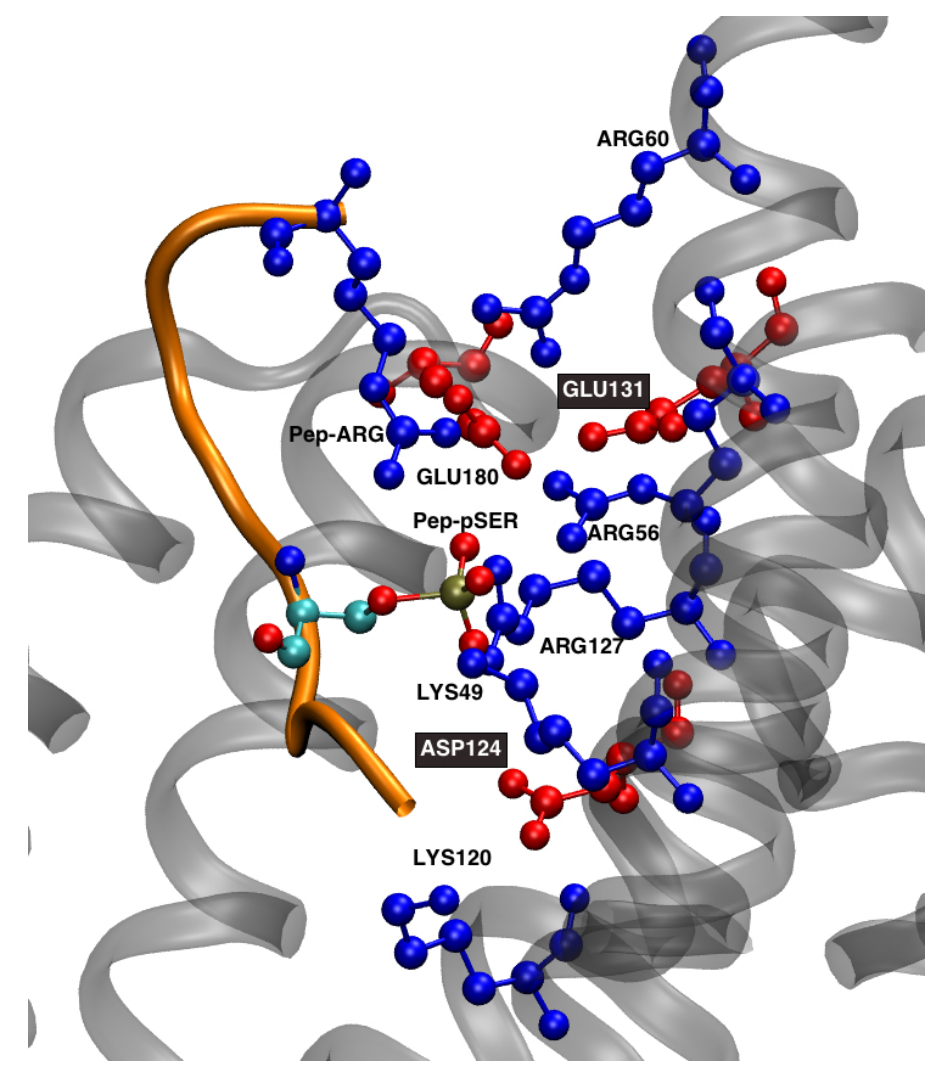

FIGURE 3 Cartoon representation of the binding pocket of14-3-3 WT M1 (PDB id: 1QJA). The bound phosphopeptide is shown in orange, charged residues in the phosphopeptide, $R$ and phosphorylated serine, $p(S)$, are shown as sticks. The interacting charged residues in the binding pocket which are within $4 \AA$ are shown in blue (basic) and red (acidic). 
(a)

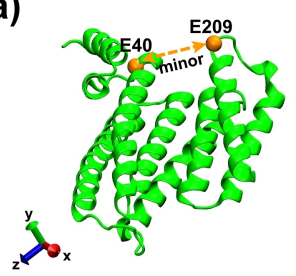

$(\mathbf{c})_{25}$

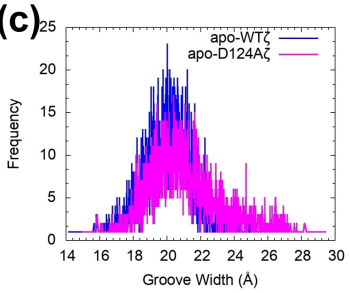

(b)

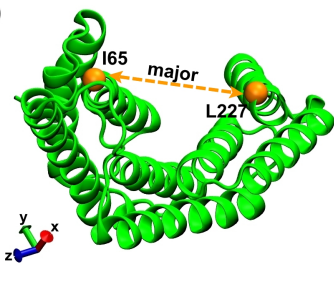

(d) 2

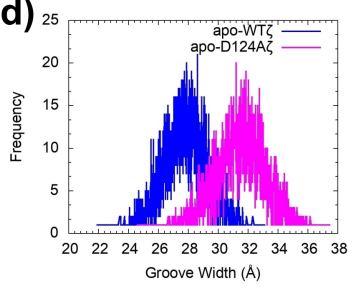

(e)

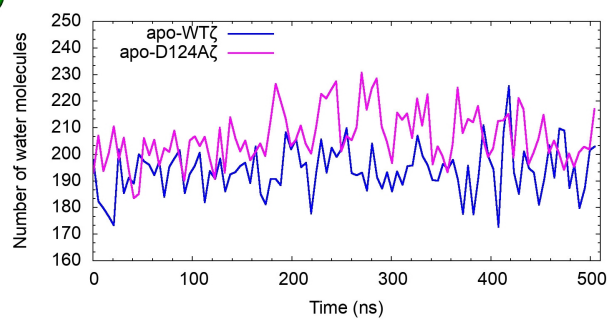

FIGURE 4 (a) cartoon representation showing minor groove defined as distance between $\mathrm{C} \alpha$ atoms of residues 40 and 209 in M1 (b) cartoon representation showing major groove defined as distance between $\mathrm{C} \alpha$ atoms of residues 65 and 227 in M1 (c) Binding pocket width distribution in M1 over the length of the simulation in minor groove (d) Binding pocket width distribution in M1 over the length of the simulation in major groove (e) Time evolution of number of waters in the binding pocket of $\mathrm{M} 1-\zeta$

(a)

(b) 159-186=EMQPTHPIRLGLALNFSVFYYEILNSPE 168-186=LGLALNFSVFYYEILNSPE

\begin{tabular}{|c|c|}
\hline Protein & $\mathbf{T}_{\mathrm{m}}$ \\
\hline WT & $63^{\circ} \mathrm{C}$ \\
\hline D124A & $50^{\circ} \mathrm{C}$ \\
\hline
\end{tabular}

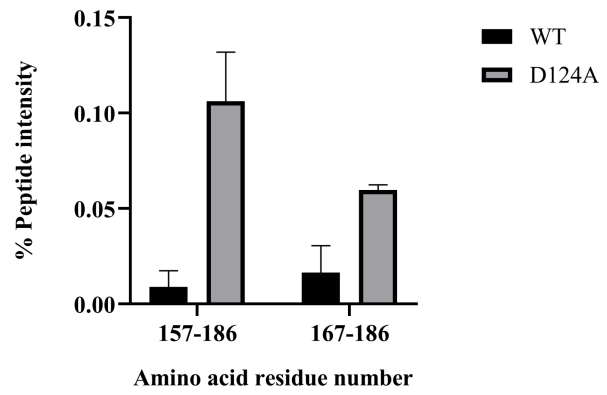

FIGURE 5 (a) $T_{m}$ of WT $\zeta$ and D124A $\zeta$ (b) All the peptide intensities (whole protein) obtained by using skyline were summed up and considered $100 \%$, followed by calculating $\%$ intensity of each peptide and plotted using the GraphPad prism. 
WT $\zeta$ and D124A $\zeta$. The data suggests that with the D124A mutation, there is an increase in the number of resident water molecules in the binding pocket(Fig. 4(e), Figure S2(c)). Taken together these results indicate that the D124A mutation causes structural rearrangement of the binding pocket making it more open and accessible, corroborating experimental observations.

\subsection{Effect of mutation on structural stability of 14-3-3:}

Although D124A mutation didn't show much change in global RMSF values, the effect on binding groove was quite evident. To validate the effect of mutation on the 14-3-3 structure observed by simulation data, thermal denaturation was monitored using intrinsic Tryptophan fluorescence as a probe and stability of the proteins was compared. $T_{m}$ of the $\mathrm{WT} \zeta$ protein was $61.8^{\circ} \mathrm{C}$ while that of D124A $\zeta$ was $50^{\circ} \mathrm{C}$ (Fig. 5 (a)) consistent with previous data published by [21]. The decrease in $T_{m}$ value indicates the loss of structural stability due to mutation.

It is possible to predict specific conformational changes in the protein using LiP-MS. In LiP-MS, the WT and D124A digested with trypsin in limited amount and products are evaluated by mass spectrometry. Obtained peptide intensities are directly proportional to the trypsin-accessible surface. When the WT and D124A peptide profiles were analysed, most of the peptide intensities were equivalent except very specific peptide spanning the region 158-186 which showed drastic increase in peptide intensities in mutant and almost undetectable in the WT (Fig. 5 (b)). This segment belongs to $\alpha \mathrm{G}$ helix, the part of amphipathic groove which harbours one of the key residues involved in the peptide binding i.e., N173. This result indicates very specific local changes in the structure that is confined to the binding pocket due to the mutation.

\subsection{Effect of D124A peptide binding amino acid residues:}

In both the monomers of WT $\zeta$ isoform , D124 interacts with other charged residues K120, R127 and N173 (Fig. 6 (a) , Figure S3(a)) . MD simulations of the apo-forms of both WT $\zeta$ and D124A $\zeta$ proteins reveal that these interactions are disrupted after the D124A mutation (Fig. 6(b-d), Figure S3(b-d)). D124 mutation brings about two changes a) conversion of charged residue to neutral residue b) replacement of bulky residue with smaller residue resulting into electrostatic and volume changes respectively. The loss of stabilising interactions between D124A mutated residue and other charged residues results in significant changes in the orientation of these residues inside the empty binding pocket (apo-form), which can have profound effect on the phosphopeptide binding.

The orientation of charged residues in the binding pockets of WT and D124A were compared in both apo and holo forms (Fig. 7(a)). In WT, R127 is closer to D124 and interacts with incoming phosphorylated serine [37, 38, 39] (Figure S1) . After D124A mutation, due to the loss of interaction with A124, this residue moves away from its WT $\zeta$ position (Fig. 7(a), Figure S4(a), shown in purple). This new conformation allows R127 to interact with another binding pocket charged residue E180 (Fig. 7(b-d), Figure S4(b-d)). To further quantify this change in conformation of R127, time evolution of dihedral angle of the residue, CG-CD-NE-CZ in M1 (Fig. 7(e)) and CB-CG-CD-NE in M2 (Figure S4(e)) was calculated, which shows a prominent change after D124A mutation in apo-WT $\zeta$.

Such conformation of R127 was observed only in D124 apo form(Fig. 7(a), Figure S4(a)), while its conformation in apo-WT $\zeta$, holo-WT $\zeta$ and holo-D124A $\zeta$ are very similar. This prompted us to ask, how this gain of interaction of R127 and E180 would affect indirect peptide binding. This difference in conformation between the apo and holo D124A $\zeta$ systems is most likley due the presence of phosphopeptide which restricts conformational freedom of R127 favoring peptide interaction. The side chain of $\mathrm{N} 173$ forms a hydrogen bond with the peptide backbone $\mathrm{NH}$ and $\mathrm{CO}$ groups of the phosphopeptide residue Leu in the mode 2 crystal structure [40,38] (Figure S1). In the simulated apo D124A $\zeta$ 
bioRxiv preprint doi: https://doi.org/10.1101/2021.09.27.461903; this version posted September 27, 2021.

The copyright holder for this preprint (which was not certified by peer review) is the author/funder. All rights

(a)
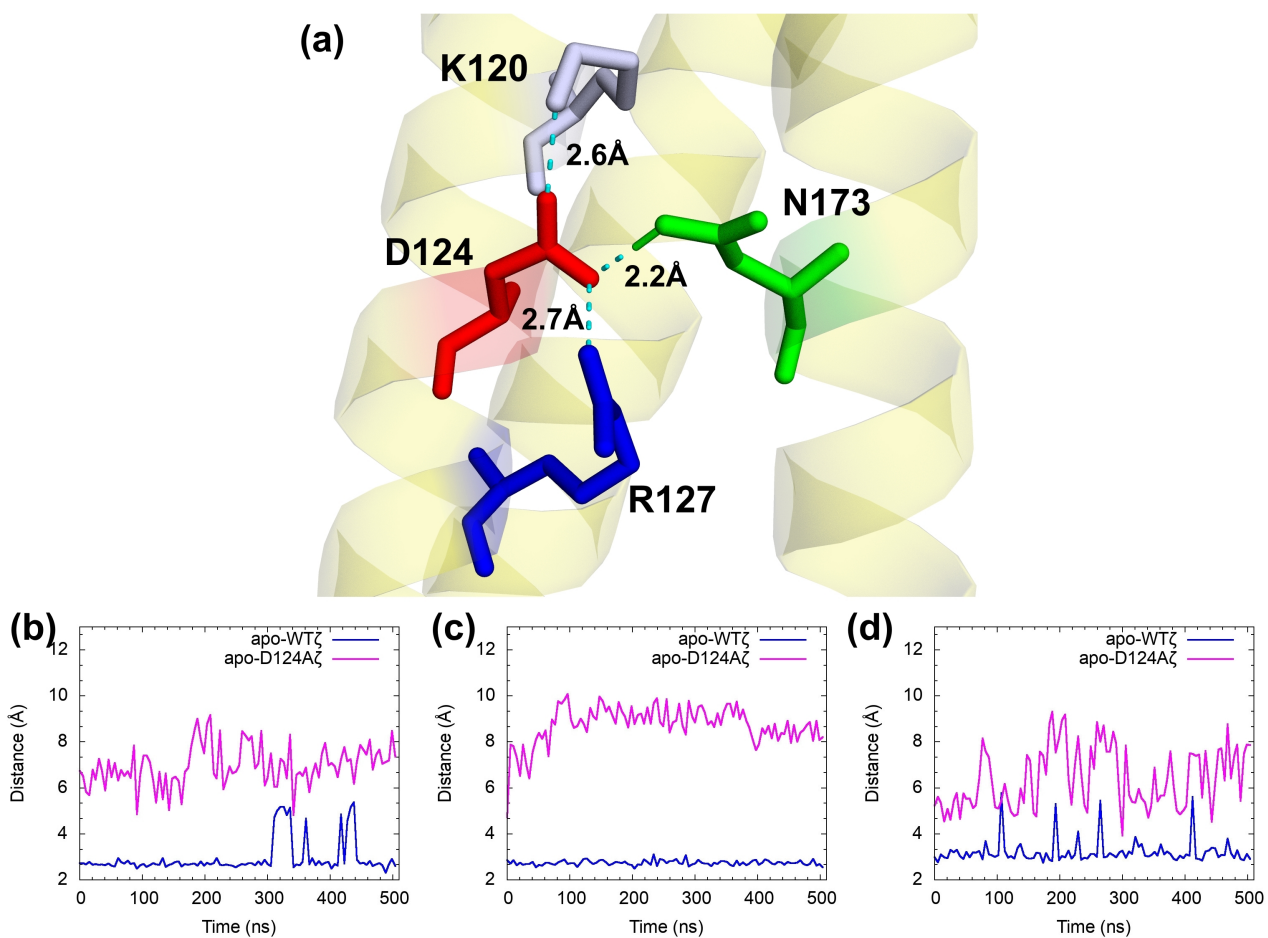

FIG URE 6 (a)D124 residue and its interacting partners K120, R127 and N173 are shown in M1(structure at end of the simulation) along with the distances between them. (b-d) Time evolution of distance plots showing the loss of interaction between D124 and K120, R127 and N173 after D124A mutation. 
bioRxiv preprint doi: https://doi.org/10.1101/2021.09.27.461903; this version posted September 27, 2021.

(a)

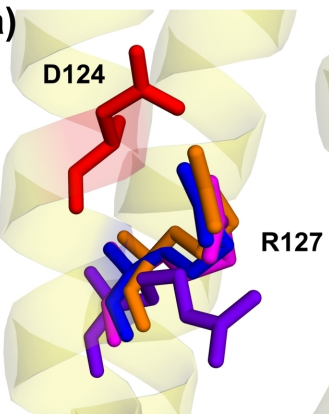

(b)

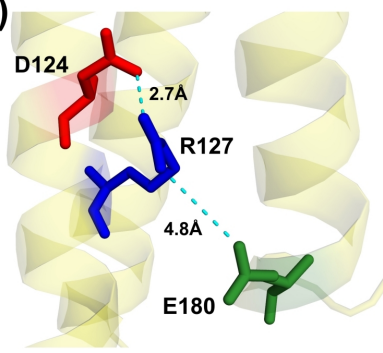

(e)

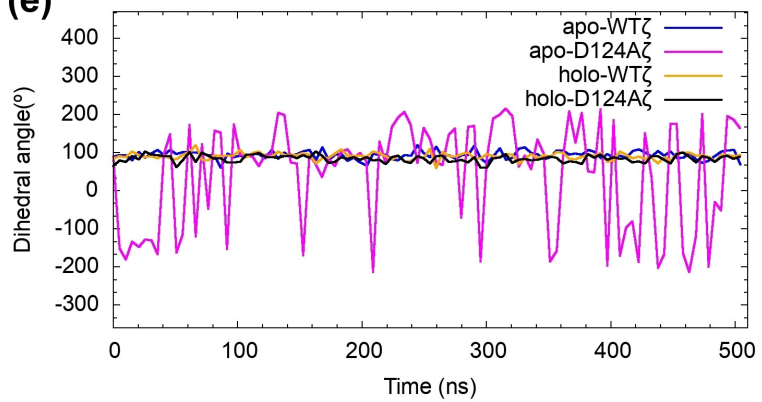

(c)

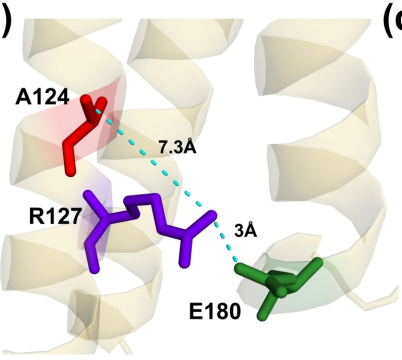

(d)

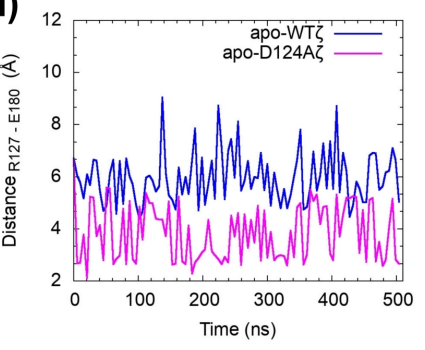

FIGURE 7 (a)change in the conformation of R127 in apo-D124A $\zeta$-M1(purple) as compared to apo-WT $\zeta$-M1

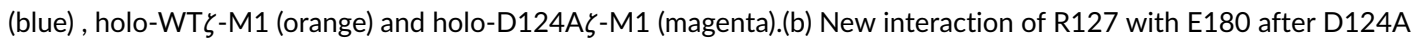
mutation ; (b)WT $\zeta$ and (c)D124A $\zeta$ in M1 (d) Distance plot showing a consistent new interaction between E180 and R127. (e) Dihedral angle CG-CD-NE-CZ changes upon D124A mutation 

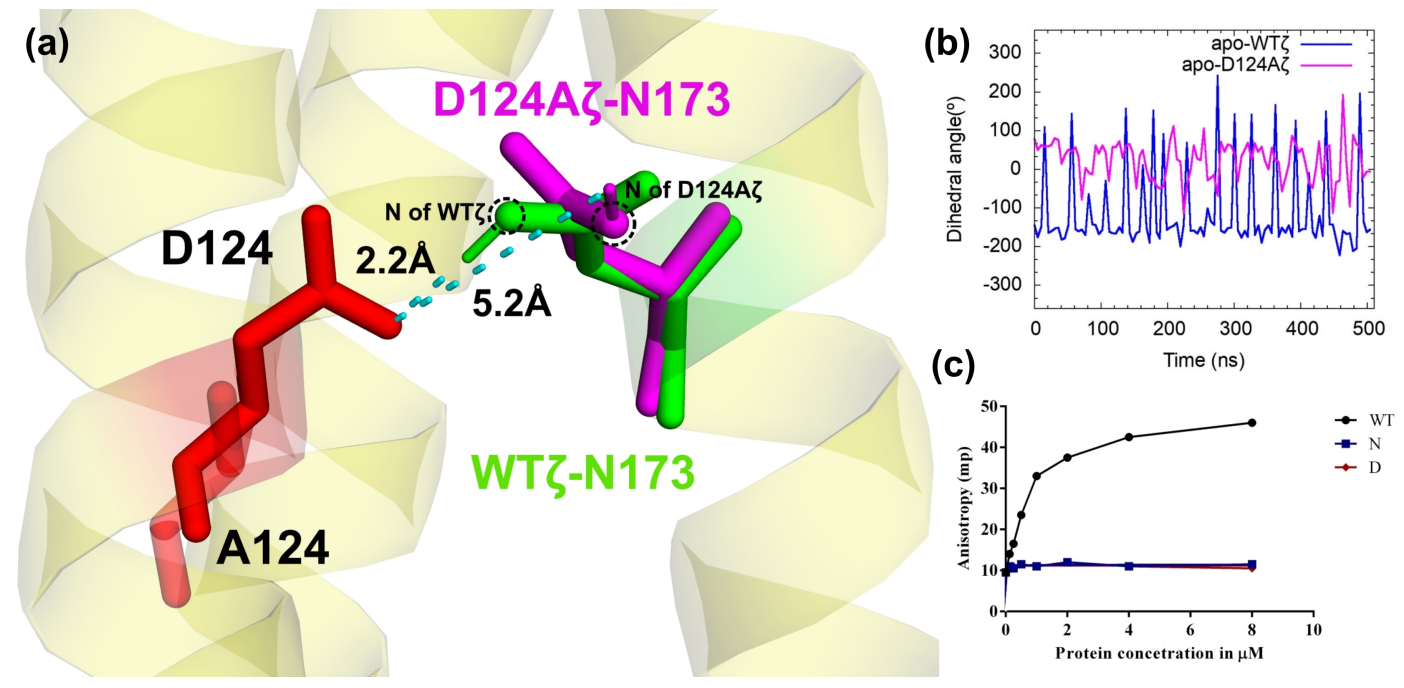

FIGURE 8 (a)change in the orientation of sidechain in N173 in apo-D124A $\zeta-M 1$ (b) The difference in the

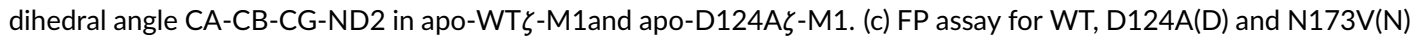

structure, the orientation of the N173 side chain also flips, resulting in loss of N173 peptide backbone contact (Fig. 8(a), Figure S5(a)). This change in conformation of N173 can have profound implications on phosphopeptide binding to the groove in apo-D124A $\zeta$ system. To further quantify the change in conformation of N173, dihedral angle CA-CB-CGND2 was calculated (Fig. 8(b), Figure S5(b)) and a distinct change in dihedral angle CA-CB-CG-ND2 was measured and confirmed the local changes in conformation caused by D124A mutation. This is notably present in only D124A-apo form and not in holo form, where the bound peptide may potential stabilize these interactions.

\subsection{4-3-3-peptide interaction and effect of mutations in vitro:}

One of the major mechanistic reason for reduction in peptide binding in D124A mutation, from MD simulations here, is the loss of peptide backbone interaction with N173. We tested peptide binding ability of N173V mutant using D124A as a positive control for reduction in peptide binding. Various protein concentrations of WT and mutant proteins were titrated against the fixed concentration of 1QJA phosphopeptide by Fluorescence polarization. N173V like D124A showed very little binding to the peptide, confirming the role of D124 in stabilizing the network of interaction at amphipathic pocket(Fig. 8(c)).

\section{4 | DISCUSSION AND CONCLUSION}

Understanding per residue contribution to protein-ligand and protein-protein interaction from structure alone is a formidable task. Nevertheless, structure is an essential guide to probe the role of dominant interactions at protein interface, which can contribute via local conformational changes, allosteric interactions in the cycle of association, dissociation to the structure and the resulting function. The 14-3-3 family of proteins are integral to the regulation mediated by post translational modifications as they sequester several 100s of phosphoproteins and release them in a precisely regulated manner to regulate a myriad of processes in the cells $[16,17,18,19]$. Crystal structures of 
many peptide bound forms and few protein bound forms are available [41, 42, 43, 44, 45] and yet answers to many of the key questions remain elusive. Some of these outstanding questions include: a) how binding is initiated b) what is the lifetime of the complex c) what are the conformational changes in the 14-3-3 or the client protein required for association and dissociation d) how does peptide and protein binding differ e) what are the secondary binding sites that stabilize the protein interaction and f) what are the different mechanisms by which of protein selectivity versus affinity is determined between the isoforms. By taking advantage of the available structures one can do specific mutations and perform extensive molecular dynamics simulations to translate the structural information into an energetic and functional map that is no longer static but captures the nuances of local geometry, dynamics and long-range conformational changes. Besides interrogating mutations and their effect on binding, an experimentalist can complement the MD studies by performing what is termed as the LIP MS. This technique helps to precisely identify the regions that undergo conformational changes over a variety of conditions including the bound versus unbound form or the WT and mutant structure.

In this study we use these two approaches to investigate what happens when an invariant negatively charged residue in the binding pocket of 14-3-3 is mutated to a neutral and small residue such as Alanine? We also asked if we could find an answer to the stark difference between phosphopeptide binding in vitro and phosphoprotein binding ex vivo when such a mutation is created. The key observations are: a) D124A mutation affects peptide binding directly by altering the geometry of the N173 which is normally engaged in a hydrogen bond interaction with the peptide back bone. Mutation of N173 results in loss of peptide binding, confirming these observations; b) D124A mutation results in widening of the entry and exit port of the amphipathic pocket which will increase the rate of dissociation. C) A bound ligand locks the geometry of the network residues in the WT like conformation explaining why we could still measure peptide binding by a more sensitive assay such as SPR. D) The accessibility of a helix 7 which packs the floor of the binding pocket in the mutant may enhance the on-rate of association of a phospho-protein. It is likely that the disordered helix in the apoform of the mutant regains its structure in the phosphoprotein bound configuration. Increase in the number of water molecules in the binding pocket, via MD simulations, further confirms the dynamic nature and increased accessibility of the binding pocket in the mutant.

With these observations in place we propose a common mechanism of mutation induced structural and conformational changes that can be extrapolated to describe the energetic map of ligand binding and common rules that distinguish peptide and protein binding across 14-3-3 isoforms. And these are: (a) mutation of D124 has profound effects on binding of the phosphopeptide; gain and loss of electrostatic interactions in the form of salt bridges strongly result in net rearrangement of the residues only in the apo form of the mutant cumulative effect of which is to weaken the interactions at the binding pocket. This observation comes exclusively from the MD simulations. (b) As predicted from our previous experimental studies on the $\gamma$ isoform [21, 15] and reiterated by investigations here on the $\zeta$ isoforms, D124A mutation induces local conformational changes and opens up the minor and major grooves that likely guard the entry and exit of the ligand; such a precise mapping is made possible by the comparative simulation of the WT and mutant proteins. (c) complementing these studies, the LIP-MS identifies a segment spanning 158-186 residues that is exclusively dynamic in the apo D124A mutant. We argue that such an opening would knock out a shorter peptide with limited area of interaction enhancing its dissociation while a large protein ligand such as seen with the 14-3-3 $\gamma$-NPM1 protein interaction, would cause 'binding induced folding' that rearranges the network of residues leading to the retention of the ligand at the binding pocket. Aspartate is invariant at this position because it is required for precise geometric orientation of interacting residues and disallowing intra protein interaction that would cost key interaction with the ligand and it is required for stability and compactness of the amphipathic groove that precisely determines the on-rate of association and off-rate of dissociation acting as a timer of binding events. Finally, we conclude that application of multiple techniques that probe atomic level interactions and account for protein dynamics 
are important for arriving at some of the basic rules of protein interactions and go beyond a static snapshot provided by crystal structure. Unduly longer association between two proteins can be as detrimental as weak protein interactions and can be potent enough to deregulate homeostasis. Evolution seems to guard cellular functions by defining amino acid conservation that appear counterintuitive such as negatively charged Asp residue in 14-3-3 very close to the strongly negatively charged phosphorylated ligand in the binding pocket. Thus by two different techniques, we map the structural and conformational changes in $14-3-3 \zeta$ in response to the mutation of an invariant negatively charged amino acid and provide plausible explanation in atomic details for the observed experimental observations.

\section{5 | ACKNOWLEDGEMENTS}

The simulations were carried out on the supercomputing machines Annapurna and Nandadevi at The Institute of Mathematical Sciences. The experimental work was funded by a grant from DST-SERB (Department of Science and Technology-Science and Engineering Regulatory Board),Government of India (EMR/2016/004079) and Intramural grant from ACTREC, Tata Memorial Centre (TMC) IRB Grant (Number 3840). ACTREC, TMC IRB Grant (Number 3840) supported Somavally Dalvi. We thank Dr. Kruti Modi for the cloning of 14-3-3 constructs. 


\section{references}

[1] Rubio MP, Geraghty KM, Barry H, CAMPBELL DG, MORRICE N, MACKINTOSH C, et al. 14-3-3-affinity purification of over 200 human phosphoproteins reveals new links to regulation of cellular metabolism, proliferation and trafficking. Biochemical Journal. 2004;379(2):395-408.

[2] Jin J, Smith FD, Stark C, Wells CD, Fawcett JP, Kulkarni S, et al. Proteomic, functional, and domain-based analysis of in vivo 14-3-3 binding proteins involved in cytoskeletal regulation and cellular organization. Current biology. 2004;14(16):1436-1450.

[3] Meek SE, Lane WS, Piwnica-Worms H. Comprehensive proteomic analysis of interphase and mitotic 14-3-3-binding proteins. Journal of Biological Chemistry. 2004;279(31):32046-32054.

[4] Benzinger A, Muster N, Koch HB, Yates JR, Hermeking H. Targeted proteomic analysis of 14-3-3 5 , a p53 effector commonly silenced in cancer. Molecular \& cellular proteomics. 2005;4(6):785-795.

[5] Dalal SN, Yaffe MB, DeCaprio JA. 14-3-3 family members act coordinately to regulate mitotic progression. Cell Cycle. 2004;3(5):670-675.

[6] Hermeking H. The 14-3-3 cancer connection. Nature Reviews Cancer. 2003;3(12):931.

[7] Hosing AS, Kundu ST, Dalal SN. 14-3-3 Gamma is required to enforce both the incomplete S phase and G2 DNA damage checkpoints. Cell Cycle. 2008;7(20):3171-3179.

[8] Aitken A. Post-translational modification of 14-3-3 isoforms and regulation of cellular function. In: Seminars in cell \& developmental biology. vol. 22. Elsevier; 2011. p. 673-680.

[9] Moore B. Specific acidic proteins of the nervous system. Physiological and Biochemical Aspects of Nervous Integration. 1967;p. 343-359.

[10] Xiao B, Smerdon SJ, Jones DH, Dodson GG, Soneji Y, Aitken A, et al. Structure of a 14-3-3 protein and implications for coordination of multiple signalling pathways. Nature. 1995;376(6536):188-191.

[11] Ichimura T, Isobe T, Okuyama T, Takahashi N, Araki K, Kuwano R, et al. Molecular cloning of cDNA coding for brainspecific 14-3-3 protein, a protein kinase-dependent activator of tyrosine and tryptophan hydroxylases. Proceedings of the National Academy of Sciences. 1988;85(19):7084-7088.

[12] Martin H, Patel Y, Jones D, Howell S, Robinson K, Aitken A. Antibodies against the major brain isofbrms of 14-3-3 protein. FEBS letters. 1993;331(3):296-303.

[13] van Hemert MJ, Steensma HY, van Heusden GPH. 14-3-3 proteins: key regulators of cell division, signalling and apoptosis. Bioessays. 2001;23(10):936-946.

[14] Rosenquist M, Sehnke P, Ferl RJ, Sommarin M, Larsson C. Evolution of the 14-3-3 protein family: does the large number of isoforms in multicellular organisms reflect functional specificity? Journal of Molecular Evolution. 2000;51(5):446458.

[15] Bose A, Modi K, Dey S, Dalvi S, Nadkarni P, Sudarshan M, et al. 14-3-3 $\gamma$ prevents centrosome duplication by inhibiting NPM1 function. Genes to Cells. 2021;.

[16] Yaffe MB, Rittinger K, Volinia S, Caron PR, Aitken A, Leffers H, et al. The structural basis for 14-3-3: phosphopeptide binding specificity. Cell. 1997;91(7):961-971.

[17] Zhao J, Meyerkord CL, Du Y, Khuri FR, Fu H. 14-3-3 proteins as potential therapeutic targets. In: Seminars in cell \& developmental biology. vol. 22. Elsevier; 2011. p. 705-712. 
bioRxiv preprint doi: https://doi.org/10.1101/2021.09.27.461903; this version posted September 27, 2021.

The copyright holder for this preprint (which was not certified by peer review) is the author/funder. All rights REFERENCES reserved. No reuse allowed without permission.

[18] Muslin AJ, Tanner JW, Allen PM, Shaw AS. Interaction of 14-3-3 with signaling proteins is mediated by the recognition of phosphoserine. Cell. 1996;84(6):889-897.

[19] Bridges D, Moorhead GB. 14-3-3 proteins: a number of functions for a numbered protein. Sci STKE. 2005;2005(296):re10-re10.

[20] Liu D, Bienkowska J, Petosa C, Collier RJ, Fu H, Liddington R. Crystal structure of the zeta isoform of the 14-3-3 protein. Nature. 1995;376(6536):191.

[21] Modi K, Dalvi S, Venkatraman P. Two negatively charged invariant residues influence ligand binding and conformational

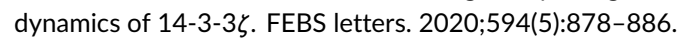

[22] Fiser A, Šali A. Modeller: generation and refinement of homology-based protein structure models. In: Methods in enzymology. vol. 374. Elsevier; 2003. p. 461-491.

[23] Tan KP, Nguyen TB, Patel S, Varadarajan R, Madhusudhan MS. Depth: a web server to compute depth, cavity sizes, detect potential small-molecule ligand-binding cavities and predict the pKa of ionizable residues in proteins. Nucleic acids research. 2013;41(W1):W314-W321.

[24] Jorgensen WL, Chandrasekhar J, Madura JD, Impey RW, Klein ML. Comparison of simple potential functions for simulating liquid water. The Journal of chemical physics. 1983;79(2):926-935.

[25] MacKerell Jr AD, Bashford D, Bellott M, Dunbrack Jr RL, Evanseck JD, Field MJ, et al. All-atom empirical potential for molecular modeling and dynamics studies of proteins. The journal of physical chemistry B. 1998;102(18):3586-3616.

[26] Best RB, Zhu X, Shim J, Lopes PE, Mittal J, Feig M, et al. Optimization of the additive CHARMM all-atom protein force field targeting improved sampling of the backbone $\phi, \psi$ and side-chain $\chi 1$ and $\chi 2$ dihedral angles. Journal of chemical theory and computation. 2012;8(9):3257-3273.

[27] Phillips JC, Braun R, Wang W, Gumbart J, Tajkhorshid E, Villa E, et al. Scalable molecular dynamics with NAMD. Journal of computational chemistry. 2005;26(16):1781-1802.

[28] Humphrey W, Dalke A, Schulten K. VMD - Visual Molecular Dynamics. Journal of Molecular Graphics. 1996;14:33-38.

[29] Schrödinger, LLC. The PyMOL Molecular Graphics System, Version 1.8; 2015.

[30] Powell MJD. Restart procedures for the conjugate gradient method. Mathematical programming. 1977;12(1):241-254.

[31] Allen MP, Tildesley DJ. Computer simulation of liquids. Oxford university press; 2017.

[32] Martyna GJ, Tobias DJ, Klein ML. Constant pressure molecular dynamics algorithms. The Journal of Chemical Physics. 1994;101(5):4177-4189.

[33] Feller SE, Zhang Y, Pastor RW, Brooks BR. Constant pressure molecular dynamics simulation: the Langevin piston method. The Journal of chemical physics. 1995;103(11):4613-4621.

[34] Darden T, York D, Pedersen L. Particle mesh Ewald: An Nlog (N) method for Ewald sums in large systems. The Journal of chemical physics. 1993;98(12):10089-10092.

[35] Essmann U, Perera L, Berkowitz ML, Darden T, Lee H, Pedersen LG. A smooth particle mesh Ewald method. The Journal of chemical physics. 1995;103(19):8577-8593.

[36] Feng Y, De Franceschi G, Kahraman A, Soste M, Melnik A, Boersema PJ, et al. Global analysis of protein structural changes in complex proteomes. Nature biotechnology. 2014;32(10):1036-1044.

[37] Fu H, Subramanian RR, Masters SC. 14-3-3 proteins: structure, function, and regulation. Annual review of pharmacology and toxicology. 2000;40(1):617-647. 
bioRxiv preprint doi: https://doi.org/10.1101/2021.09.27.461903; this version posted September 27, 2021.

[38] Rittinger K, Budman J, Xu J, Volinia S, Cantley LC, Smerdon SJ, et al. Structural analysis of 14-3-3 phosphopeptide complexes identifies a dual role for the nuclear export signal of 14-3-3 in ligand binding. Molecular cell. 1999;4(2):153166.

[39] Yaffe MB. How do 14-3-3 proteins work?-Gatekeeper phosphorylation and the molecular anvil hypothesis. FEBS letters. 2002;513(1):53-57.

[40] Yang X, Lee WH, Sobott F, Papagrigoriou E, Robinson CV, Grossmann JG, et al. Structural basis for protein-protein interactions in the 14-3-3 protein family. Proceedings of the National Academy of Sciences. 2006;103(46):1723717242.

[41] Stevers LM, Lam CV, Leysen SF, Meijer FA, van Scheppingen DS, de Vries RM, et al. Characterization and small-molecule stabilization of the multisite tandem binding between 14-3-3 and the R domain of CFTR. Proceedings of the National Academy of Sciences. 2016;113(9):E1152-E1161.

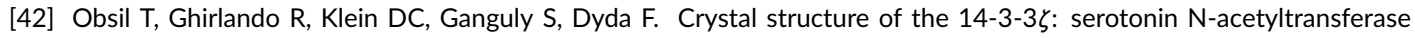
complex: a role for scaffolding in enzyme regulation. Cell. 2001;105(2):257-267.

[43] Sluchanko NN, Beelen S, Kulikova AA, Weeks SD, Antson AA, Gusev NB, et al. Structural basis for the interaction of a human small heat shock protein with the 14-3-3 universal signaling regulator. Structure. 2017;25(2):305-316.

[44] Benzinger A, Popowicz GM, Joma KJ, Majumdar S, Holak TA, Hermeking H. The crystal structure of the nonliganded 14-3-3 $\sigma$ protein: insights into determinants of isoform specific ligand binding and dimerization. Cell research. 2005;15(4):219-227.

[45] Borisova ME, Voigt A, Tollenaere MA, Sahu SK, Juretschke T, Kreim N, et al. p38-MK2 signaling axis regulates RNA metabolism after UV-light-induced DNA damage. Nature communications. 2018;9(1):1-16. 